\title{
On the Recirculation Zone Behind a Cylinder of Finite Length with Subcritical Aspect Ratio
}

\author{
Pavel Procházka ${ }^{1, *}$, and Václav Uruba ${ }^{1,2}$ \\ ${ }^{1}$ Institute of Thermomechanics, ASCR, v.v.i., Dolejškova 5, Praha 8, CR \\ ${ }^{2}$ UWB, Faculty of Mechanical Engineering, Department of Power System Engineering, Universitní 8, \\ Plzeň, CR
}

\begin{abstract}
The Particle Image Velocimetry is utilized to investigate flow behind a cylinder of finite length with subcritical value of Aspect Ratio (AR). The cylinder is submitted to uniform freestream with very low intensity of turbulence and thin laminar boundary layer. The flow field cannot be expected as 2D even for time-averaged quantities. The Strouhal number is evaluated in various planes of measurement. The wake is fully turbulent and is composed of many streamwise and spanwise-oriented structures. Such complex flow is very convenient to be subjected to dynamical decomposition analysis.
\end{abstract}

\section{Introduction}

The infinite circular cylinder belongs to the classical canonical cases of the flow field behind bluff-bodies. One of the first explorers which were dealing with the flow around the infinite cylinder was Strouhal and von Kármán. However, a first comprehensive publication was published in the fifties by Roshko [1]. To this day many experimental as well as numerical scientific papers (e. g. [2]) were published and the gained pieces of knowledge are utilized for many industrial and building industry applications (struts, antennas, pipes, marine cables etc.). In fact, many of them are in finite length shape as various chimneys, cooling towers or buildings. A free end of the cylinder affects the near wake behind such obstacle significantly. It was shown that critical property is Aspect Ration $A R=L / D$ as a ratio of the length to outer diameter.

For short circular cylinder (AR < 7), von Kármán vortex street is effectively suppressed by impact of the recirculating wake flow. The motivation of this research paper is to examine the typical topology of the circular cylinder wake with critical Aspect Ratio from statistical as well as dynamical point of view.

This phenomenon is solved by many research groups worldwide, especially by numerical simulations. Experimental research is very often devoted to finite-length cylinders with rectangular geometry [3] which is an area of particular interest to architects. Also, there are many work dealing with circular cylinders in the literature, however, the geometry for rather high AR is tested [4]. Hölscher et al. [5] describes a critical value of AR to approximately 2.5 (usually it is reported between 2 and 6). The flow field

\footnotetext{
*Corresponding author: prochap@it.cas.cz
} 
topology passes from von Kármán vortex street form to symmetric arch-type vortex under this value (see Figure 1). Classical vortex shedding behind infinite cylinder was experimentally investigated recently [6]. The arch-type vortex is created as a consequence of the flow structures separated from flanges which interacts with the vortices separated from trailing top edge. The horse-shoe vortex is created as consequence of the boundary layer-cylinder shape interaction. Finally trailing (base) vortex is the result of strong downwash effect in the cylinder wake.

This experimental work should reveal some preliminary results from time-resolved PIV measurement conducted in many horizontal Planes of Measurement (PoM) and one plane oriented perpendicularly to the flow. The tested cylinder has subcritical Aspect Ratio equal to two, it is stuck out from bottom side of a tested channel. Statistical as well as dynamical features of the flow will be discussed which should reveal the development of tip vortical structures, trail vortices and mainly arch-type vortex. The extent of the near-wake recirculation zone and the downwash phenomenon will be shown.

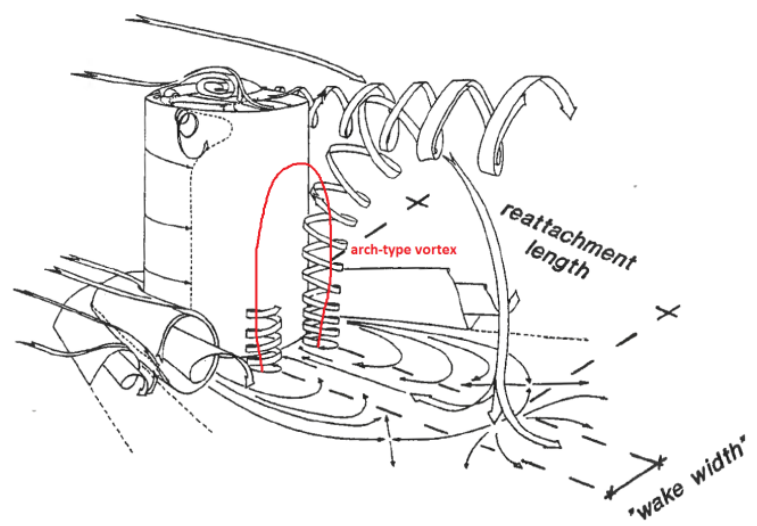

Fig. 1. 3D flow pattern and schematic view of arch-type vortex, borrowed from [5].

\section{Experiment description}

The tested cylinder had Aspect Ratio AR $=2$ (the diameter was $30 \mathrm{~mm}$ and height was $60 \mathrm{~mm}$ ). The cylinder was attached to the bottom side of the rectangular test section connected to the blow-down wind tunnel (Figure 2). The test section of cross-section dimensions $250 \times 250 \mathrm{~mm}$ was made of Perspex walls allowing optical access for PIV measurement. The Cartesian coordinate system ( $\mathrm{x}, \mathrm{y}, \mathrm{z}$ ) was established so that $\mathrm{x}$ coordinate is in streamwise direction while $\mathrm{z}$ coordinate is parallel to cylinder axis. The origin is placed in the cylinder base centre. The blockage factor caused by the model is less than $3 \%$. The incoming velocity was set to $5 \mathrm{~m} / \mathrm{s}$ and corresponding Reynolds number is 9900 . The boundary layer thickness is insignificant $(2-3 \mathrm{~mm})$ since the model is placed just behind the tunnel contraction (42 $\mathrm{mm}$ downstream).

Time-resolved Particle Image Velocimetry (TR PIV) was used to measure velocity vector field. Two different approaches were utilized. Horizontal PoMs were acquired by 2D2C (two velocity component evaluated in a plane) technique which requires only one camera. On the other hand, stereo PIV (2D3C) enables to evaluate all three velocity components in a plane of measurement at the cost of using two cameras. This second approach was applied for perpendicular plane located $55 \mathrm{~mm}(\mathrm{x} / \mathrm{D}=1.83)$ downstream. The measurement apparatus consisting of a laser and cameras is from Dantec company. Laser New Wave Pegasus Nd:YLF has double head emitting coherent light with wavelength of $527 \mathrm{~nm}$, maximal frequency is $10 \mathrm{kHz}$, shot energy is $10 \mathrm{~mJ}$ (for $1 \mathrm{kHz}$ ) 
and corresponding power per head is $10 \mathrm{~W}$. The CMOS camera Phantom V611 with acquisition frequency of 3000 double-snaps per second was used as well as two VEO 410 cameras with frequency of 2500 double-snaps were used. All three cameras had resolution of 1280 x 800 pixels. The internal memory was 8 GB and 16 GB, respectively. Scheinpflug mounting was utilized for stereoscopic measurement. Safex particles with mean diameter of $1 \mu \mathrm{m}$ were used. The data were acquired and processed in DynamicStudio software, Matlab and Tecplot software.

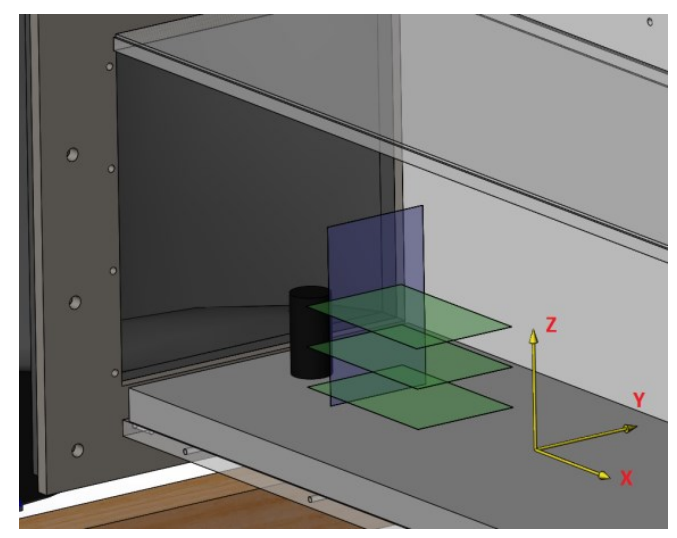

Fig. 2. The setup of a finite circular cylinder inside the test section, coordinate system and planes of measurements; blue color for cross-sectional and green color for horizontal ones.

Proper Orthogonal Decomposition (POD) was applied to extended dataset (complex wake flow field) to extract several orthonormal modes (consisting of topos and chronos) which are order according to their relative kinetic energy content. Low-order modes are characterized by higher amount of kinetic energy and contain bigger coherent structures. High-order modes have much less energy because they are created by tiny vortices and very often by only noise. Further information about decomposition methods can be found in Uruba et al. [7].

\section{Results}

All coordinates are plotted in dimensionless form (divided by cylinder diameter), also velocity value is normalized by the incoming velocity $U e=5 \mathrm{~m} / \mathrm{s}$. Further in all diagrams, black vectors represent in-plane velocity component, while the color represent the scalar value (streamwise vel. component, vorticity etc.). The blue lines are used to make visible vector lines of time-averaged flow field and green lines are isolines of vorticity. The position of circular cylinder is portrayed by a black circle (dashed black lines represents a projection of cylinder contour). An investigation of the near-wake region was performed in 13 horizontal planes $(z=3-100 \mathrm{~mm})$. Presented results will be plotted only for $z=10,30,60$ and $80 \mathrm{~mm}$, respectively $z / D=0.3,1,2$ and 2.7 in this paper. Only one cross-sectional plane in $x / D=1.83$ will be discussed here.

\subsection{Horizontal planes}

The flow field is symmetrical only for time-averaged values (Figure 3a). The black contour depicts the back-flow border and color represents streamwise velocity component. 

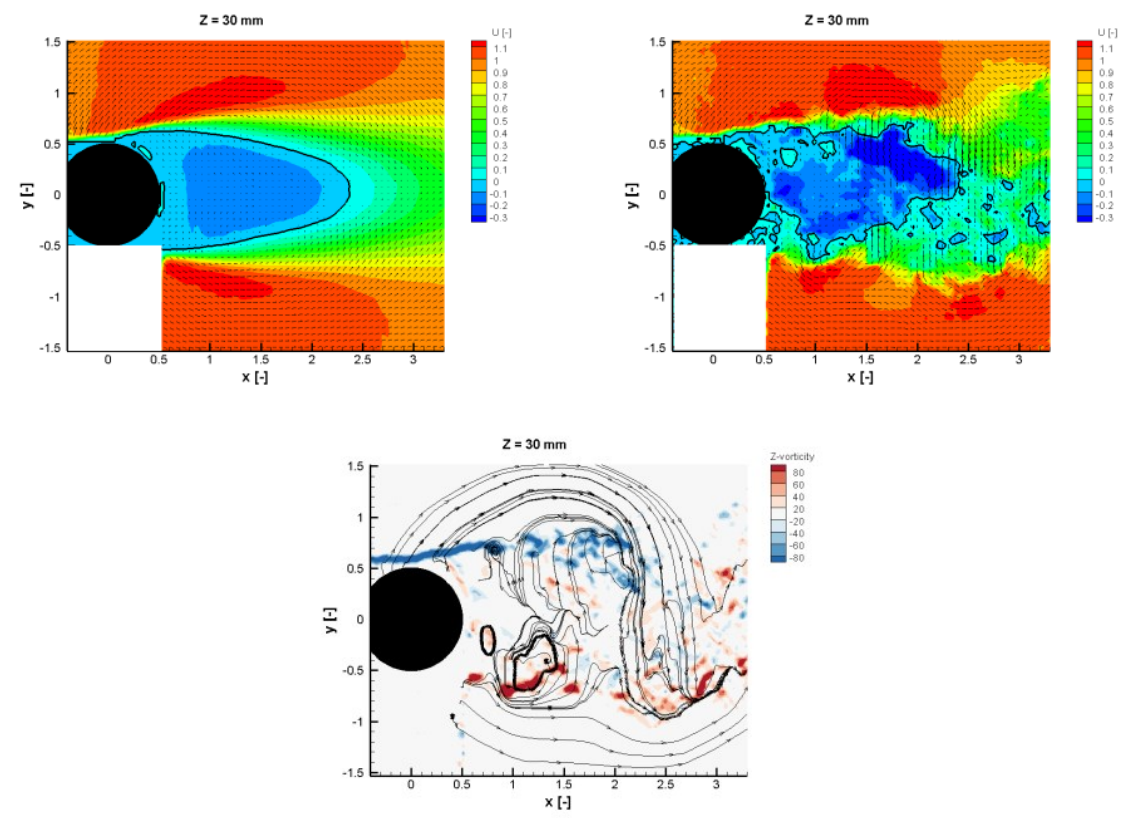

Fig. 3a, b, c. Mean flow fields and snapshot for plane $z / c=30 \mathrm{~mm}$; u-vel. component and vorticity.

Snapshot (Figure 3b) reveals that the flow behind the cylinder is characterized by high dynamical activity. Instantaneous velocity vector maps are characterized by many vortical structures in chaotic manner and high asymmetry. Fluctuation activity is high especially close to boundary of recirculation zone $y / D=+/-0.5$. On the other hand, the minimal value of TKE (turbulent kinetic energy) is right behind the cylinder leeward side. Notice that the data are incomplete due to laser illumination from the right side. Next figure is plotted for vorticity distribution and streamlines are added to visualize instantaneous features of the wake. It reveals two big eddies which were shed from the cylinder round body and forced deflected freestream. Notice the eddies are not in zig-zag pattern.

Unlike the infinity cylinder wake flow, here the mean flow field is not 2D anymore as the back-flow region extent differs for all horizontal PoMs (Figure 4a). Black, red, orange, yellow, green and blue line is used for $z=5,10,20,30,40$ and $50 \mathrm{~mm}$, respectively. There is also a small separation bubble above the cylinder free end. The curved leading edge forms a separation resulting in two counter-rotating vortices. Due to the turbulent momentum flux, the flow reattaches with strong pressure recovery [5]. The extent LW of the back-flow area is decreasing with second power as it can be seen in Figure $4 \mathrm{~b}$.
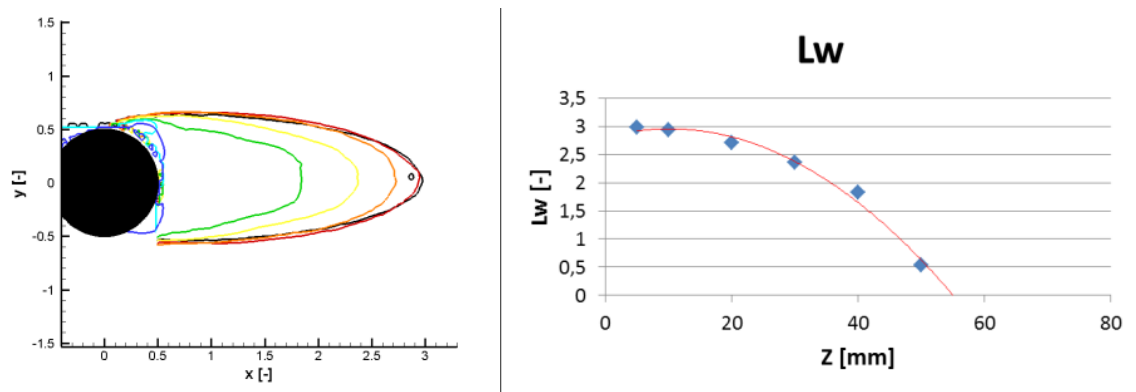

Fig. 4a, b. A comparison of wake extent Lw at different heights, $\mathrm{z}$ coordinate varies from 5 to $50 \mathrm{~mm}$. 
Quasi vortex street shedding occurs only for cylinders with AR higher than a critical value. And even so, the Strouhal number is reduced compared to an infinite cylinder. Such kind of very short cylinder is characterized by even smaller value of Sh. A frequency spectrum was calculated at distinct point of the wake for lateral velocity component. A signal of power spectral density (PSD) was very weak which implies that von Kármán vortex street is not present for subcritical AR. The Strouhal number evaluated on several horizontal planes demonstrates values between 0.14 and 0.16 (Table 1).

Table 1. Strouhal number value evaluated at several heights and corresponding frequency.

\begin{tabular}{|c|c|c|c|c|c|c|c|}
\hline $\mathbf{Z}(\mathbf{m m})$ & 3 & 5 & 10 & 15 & 20 & 30 & 40 \\
\hline $\mathbf{f ~ ( H z )}$ & 23.9 & 25.9 & 23.9 & 26.9 & 23.7 & 23.9 & 24.4 \\
\hline Sh (-) & 0.14 & 0.16 & 0.14 & 0.16 & 0.14 & 0.14 & 0.15 \\
\hline
\end{tabular}

Figure 5 shows vector line of mean flow field for four horizontal planes. Actually, the pictures are plotted as cuts of arch-type vortex. Two counter-rotating vortices create the platform of such structure (5a). In the middle of the cylinder $(30 \mathrm{~mm})$, the arch-type vortex starts to bend to horizontal orientation. The plane aligned with cylinder free end $(5 c)$ is characterized by only deflected vector lines. Strong downwash effect is here apparent (Figure 8 ). The velocity field above the cylinder body is deflected only slightly.
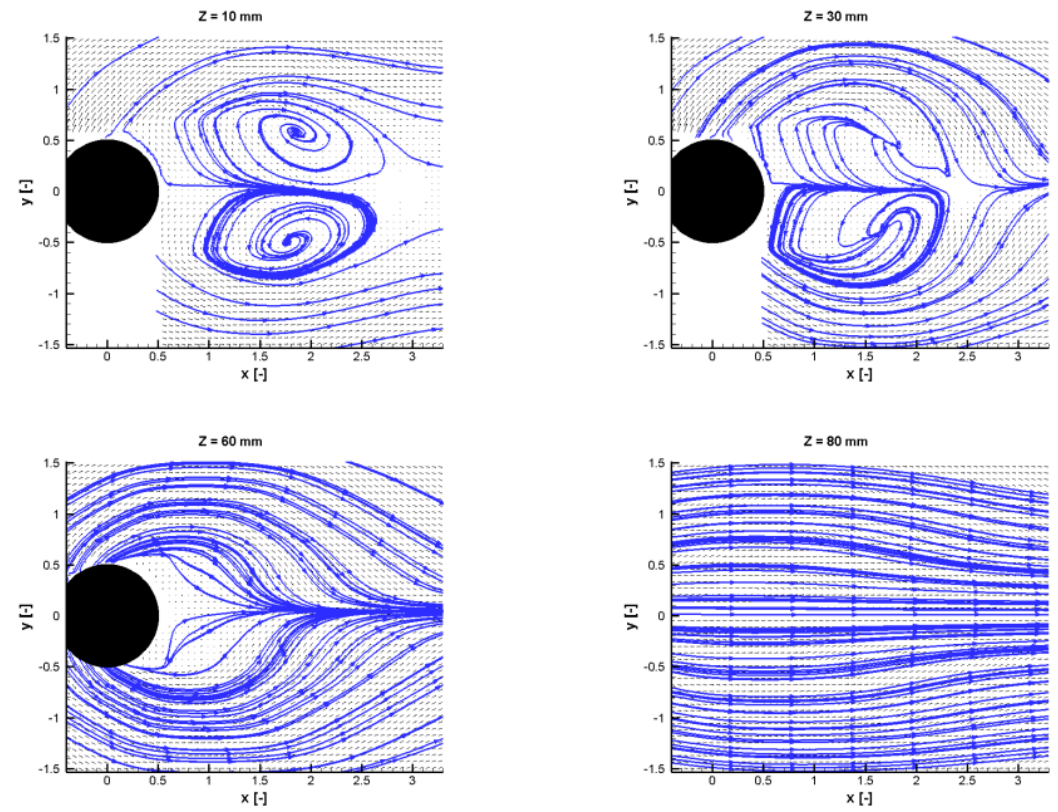

Fig. 5a, b, c, d. Mean flow fields and vector lines at four distinct planes indicating the topology of the arch-type vortex shedding.

POD decomposition was applied to flow data at 10,30 and $60 \mathrm{~mm}$ above the bottom side. The energy fraction of low-order modes is plotted in Figure 6 . The first mode with highest energy $(>20 \%)$ was detected for the very first PoM. The topos of this mode is characterized by two co-rotating vortices (counter-rotating couple is present in topos of fourth mode) at location of arch-type vortex and by one big eddy further downstream in 
the wake. A similar structure can be evaluated from the first mode in the middle plane; now strong shear flow is apparent instead the big eddy. The last example is for the upper plane aligned with cylinder top. Two pairs of strong shear layers can be observed just behind the cylinder trailing edge. These structures are obviously related to two tip vortices.

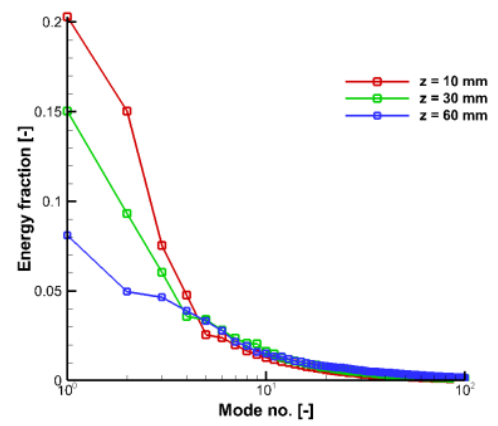

Fig. 6. The kinetic energy fraction of the first 100 POD modes at three distinct horizontal planes.
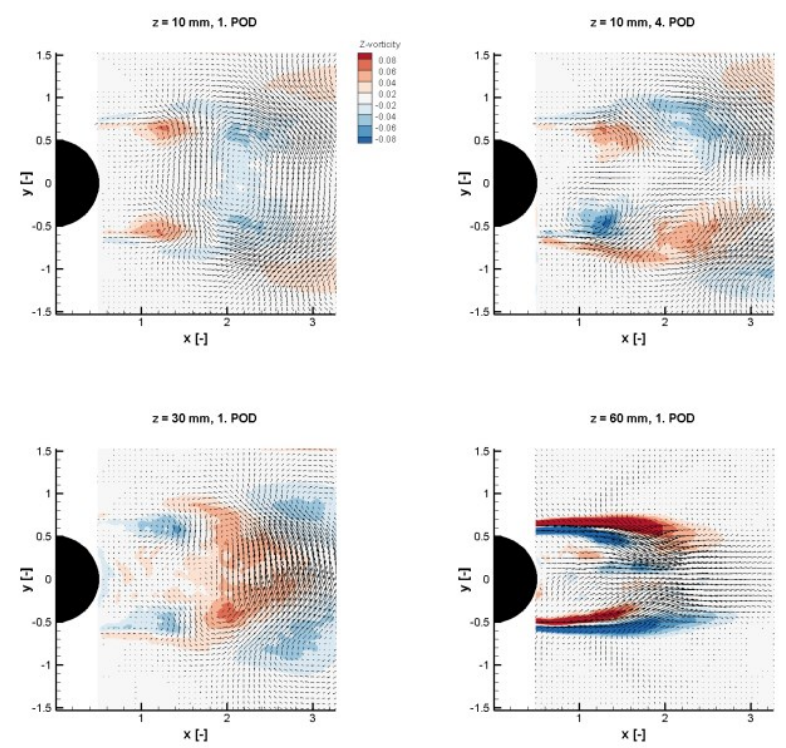

Fig. 7a, b, c, d. 1. (a, c, d) and 4. (b) POD mode for $\mathrm{z}=10 \mathrm{~mm}$ (a, b), $30 \mathrm{~mm}$ (c) and $60 \mathrm{~mm}$ (d).

\subsection{Cross-sectional plane}

The perpendicular planes measured by stereoscopic PIV technique will be studied in detail during future research activities, nevertheless results from one PoM will be presented here. This plane is located $55 \mathrm{~mm}$ downstream from the cylinder centre and represents the backflow region covering approximately $2 / 3$ height of the cylinder (see Figure $8 \mathrm{a}$ ). Very strong downwash effect is apparent, the flow is attracted from both sides as well as from above. Maximal fluctuation activity can be proved in the lower part from both sides of cylinder silhouette (this is the location of counter-rotating trailing vortices further downstream). Figure $8 \mathrm{c}$, d shows a snapshot which is characterized by strong streamwise-oriented vortex whose location is right between both legs of arch-type vortex. 
This dataset was also subjected to POD analysis. The very first mode (energy $17 \%$ ) is characterized by two significant shear layer down along the sides of the cylinder. Significant vortical structures are present in fourth mode (energy $5 \%$ ). The place between two counter-rotating vortex pair is filled by fluid of accelerating or decelerating tendency which are usually called streaks. The high-order modes topology is created by high amount of tiny vortices as it is shown in Figure 9 d.
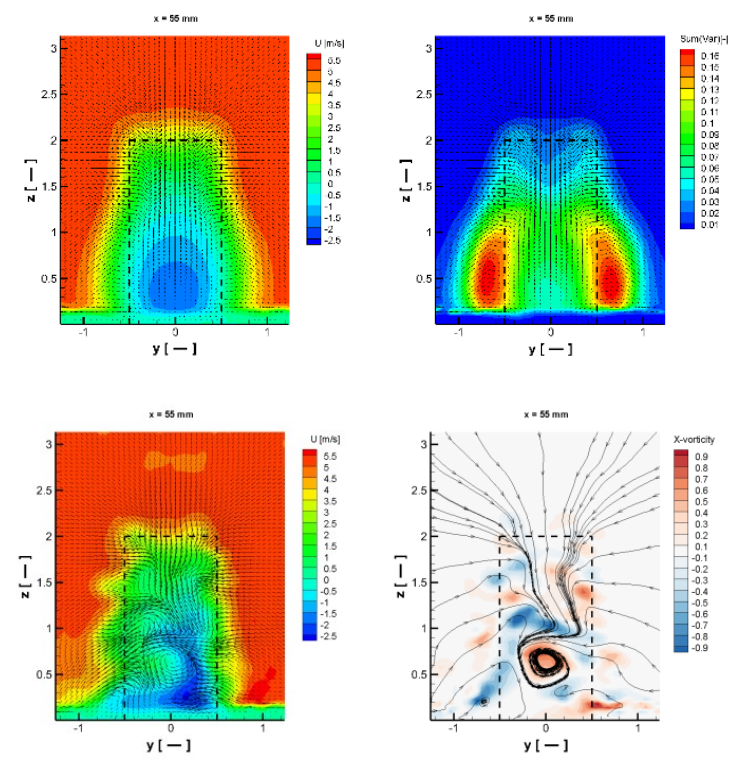

Fig. 8a, b, c, d. Mean flow fields and snapshots for plane $x=55 \mathrm{~mm}$; u-vel. component, TKE and vorticity.
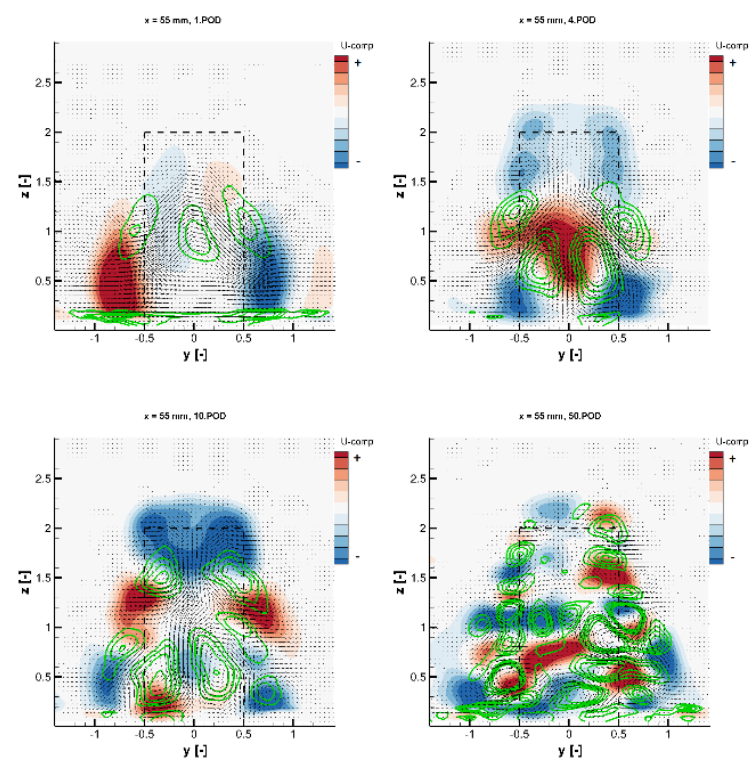

Fig. 9a, b, c, d. 1., 4., 10. and 50. POD mode evaluated at $x=55 \mathrm{~mm}$; u-vel. component distribution and vorticity isolines. 


\section{Conclusions}

This experimental work is devoted to examination of near-wake region behind short circular cylinder with smooth wall. The Aspect Ratio (AR) is subcritical, symmetric archtype vortex shedding was detect by means of TR-PIV technique. The mean flow parameters and POD decomposition was applied to acquired data. These experiments was also conducted to verify the calculation based on Reduced Order Modelling (ROM).

The size of back-flow zone was evaluated in several horizontal planes. The tip vortices and arch-type vortex and trailing vortex were detected from velocity vector fields. Very strong downwash effect was observed which significantly reduces the extent of backflow. The Strouhal number was evaluated from peak frequency in frequency spectrum. The value is in a good agreement with findings of other authors [4]; $S h=0.14-0.16$.

In the future articles, the topology of near-wake region will be reconstructed from 2D3C data acquired in several cross-sectional planes and also more advanced dynamical analysis will be performed (OPD). The effect of different value of AR and different roughness will be tested also. The dynamical behaviour of quasi-static arch-type vortex will be investigated.

This work was supported by the Grant Agency of the Czech Republic, project No. 17-01088S, 1904695S and 19-02288J.

\section{References}

1. A. Roshko, "On the development of turbulent wakes from vortex streets", NACA Report 1191 (1953)

2. M. A. Mendez, D. Hess, B. B. Watz, J.-M. Buchlin, Multiscale Proper Orthogonal Decomposition (mPOD) of TR-PIV data - a case study on stationary and transient cylinder wake flows, Measurement Science and Technology, 31, 9 (2020)

3. S. Peng, H. Wang, L. Zeng, X. He, Low-frequency dynamics of the flow around a finite-length square cylinder, Experimental Thermal and Fluid Science, 109, 109877 (2019)

4. D. Sumner, J. L. Heseltine, O. J. P. Dansereau, Wake structure of a finite circular cylinder of small aspect ratio, Experiments in Fluids, 37, 720-730 (2004)

5. N. Hölscher, H.-J. Niemann, On the flow around finite circular cylinder in turbulent shear flows, Physics of Separated Flows - Numerical, Experimental and Theoretical Aspects. Notes on Numerical Fluid Mechanics, 40 (1993)

6. P. Procházka, V. Uruba, Reynolds number effect on velocity field and on coherent structures behind a cylinder, AIP Conference Proceedings, 2118, 030037(2019)

7. V. Uruba, Decomposition methods in turbulent research, EPJ Web of Confere nces, 25, 01095 (2012) 